\title{
Pyroelectric effect enhancement through product property under open circuit condition
}

\author{
H. H. S. Chang ${ }^{\text {a) }}$ and Z. Huang \\ Department of Materials, Cranfield University, Bedfordshire, MK43 OAL, United Kingdom
}

(Received 20 January 2009; accepted 30 May 2009; published online 6 July 2009)

\begin{abstract}
An analytical model for the pyroelectric (PY) effect under open circuit condition and 2-2 connectivity laminates of various pairs of PY and nonpyroelectric (NP)/elastic materials has been developed. It is evident from our analysis that there indeed is a substantial dissimilarity between the PY coefficients and figure of merit for efficiency for various PY-NP pairs under short circuit and open circuit conditions. We believe this implies that there should be a greater distinction made between the PY coefficients under these two electrical conditions than previously thought. The indicators for various PY-NP material pairs that can be utilized to determine their PY coefficient enhancement potential under open circuit condition have been identified. The investigated PY materials are lead zirconate titanate (PZT-5H and PZT-5A), barium titanate, lithium tantalate, lithium niobate, and polyvinylidene fluoride (PVDF), while the NP materials are stainless steel, polytetrafluoroethylene (PTFE or Teflon), chlorinated polyvinyl chloride thermoplastic (CPVC), aluminum, zinc, and Invar 36. Extraordinarily large PY coefficient of $97 \times 10^{-4} \mathrm{C} \mathrm{m}^{-2} \mathrm{~K}^{-1}$ at minimum thickness ratio $R_{\min }$ is expected for PZT-5H-CPVC pair while PVDF-CPVC could show increase in the secondary PY coefficient of up to $350 \%$. In addition, where the figure of merit for efficiency is concerned, for the same volume of the composite PZT-5A-PTFE pair it reaches 24, a 24-fold increase in efficiency at $R_{\min }$. Our analysis techniques should provide a methodological way for appraising the potentials of particular PY material and its 2-2 laminates for applications under open circuit condition such as PY X-ray generation, electron accelerator, and nuclear fusion.
\end{abstract}

(C) 2009 American Institute of Physics. [DOI: 10.1063/1.3158472]

\section{INTRODUCTION}

It is well established that the pyroelectric coefficient (PY coef.), usually measured at constant stress $\mathbf{p}^{T, E}$, consists of the primary PY coef. at the constant strain $\mathbf{p}^{S, E}$ and the secondary PY coef. arising from strain, ${ }^{1}$

$$
p_{m}{ }^{T, E}=p_{m}{ }^{S, E}+d_{m k l}{ }^{E, \Theta} c_{i j k l}{ }^{E, \Theta} \alpha_{i j}{ }^{T, E} .
$$

Here, $T$ is the stress, $E$ is the electric field, $\Theta$ is the temperature, $p_{m}{ }^{T, E}$ is the PY coefficient at constant stress (free boundary condition) and electric field, $d_{m k l}^{E, \Theta}$ is the piezoelectric constant, $c_{i j k l}^{E, \Theta}$ is the elastic stiffness at constant temperature and electric field, and $\alpha_{i j}{ }^{T, E}$ is the thermal expansion coefficient at constant stress and electric field. Notice the Einstein summation convention is used throughout this article along with the Voigt notation and the secondary PY effect can be regarded as a product property of piezoelectricity and thermal expansion. ${ }^{2}$

In our other communication, ${ }^{3}$ with Eq. (1) in mind, the laws of thermodynamics dictated the PY coefficient for 2-2 laminates under short circuit (SC) condition to be

${ }^{a)}$ Electronic mail: h.chang@cranfield.ac.uk.

$$
P_{m}=\frac{d D_{m}}{d \Theta}=p_{m}{ }^{T, E}-\left(d_{m k l}^{E, \Theta}\right)\left(c_{i j k l}^{E, \Theta}\right)\left[\alpha_{i j}^{T, E}-\frac{d S_{i j}}{d \Theta}\right],
$$

where $p_{m}$ is the PY coefficient, $D_{m}$ is the electric displacement, and $d S_{i j}$ is the total strain experienced by the PY material.

This extended version of the PY coefficient expression, Eq. (2), describes the pyroelectricity of any PY material experiencing strain under SC condition. However, in practice, some applications such as PY X-ray generation, ${ }^{4-6}$ electron accelerator, ${ }^{7}$ and nuclear fusion ${ }^{8}$ are conducted under conditions most closely approximated by open circuit (OC). We believe there is a difference between the PY coefficient under OC and SC conditions, and it is paramount that this disparity between the two be formulized to provide better insights into these applications of pyroelectricity. We also believe that this PY coefficient $p^{T, D}$ under OC condition is the one that should be utilized when evaluating the "voltage figure of merit" $F_{V}$ and hence be distinguished from $p^{T, E}$ used in "current figure of merit" $F_{I}{ }^{9}$

Under SC condition, the electric potential on the whole of the surface of the crystal is perceived as being the same, in other words $\mathbf{E}$ is assumed to be zero and constant, i.e., $\mathbf{E}$ $=0=d \mathbf{E}$. Sometimes also termed as electrically free, this is the condition under which most measurements of PY coefficient are taken and therefore is the condition conventionally used for the derivation of PY coefficient, including Eq. (2). Under OC condition, however, $\mathbf{D}$ is assumed to be constant 
in a crystal. This implies $d \mathbf{D}=\varepsilon_{0} d \mathbf{E}+d \mathbf{P}=0$, where $\mathbf{P}$ is polarization, and hence the need for a different PY coefficient expression from that of Eqs. (1) and (2). In general, when $\mathbf{D}=0$ the crystal is said to be electrically clamped.

In a previous communication, we reported a substantial PY coefficient enhancement in laminar stainless steel/lead zirconate titanate (PZT)/stainless steel structures, ${ }^{3}$ with both experimental observation and theoretical prediction demonstrating PY coefficient enhancements of more than $100 \%$ under SC condition. We believe the employment of 2-2 connectivity configuration in pyroelectricity under $\mathrm{OC}$ condition also requires further exploration which could potentially uncover PY coefficient alterations of great proportions. The findings of this investigation are presented in this article.

At the outset in Sec. II, we first examine the theoretical and mathematical background behind this by applying thermodynamic and plate theory to the OC condition, results of which are presented in later section with the use of Mathematics package MAPLE $9.50 .{ }^{10}$ In order to analyze the effects of increased thermal mass due to the introduction of NP elastic layer, we also define a quantity termed efficiency and utilize it to assess the trade-off between thermal budget and increased PY response by the means of figure of merit for efficiency, namely, $F_{\text {eff }}^{a}$ and $F_{\text {eff }}^{b}$. The choice of materials and the reasons behind this preference are presented, while the outcomes of the ensuing mathematical simulations and corresponding conclusions are demonstrated in Secs. III and IV.

\section{GENERAL THEORETICAL ANALYSIS}

\section{A. Pyroelectric coefficient under OC condition}

In order to enable the mathematical model to describe our PY coefficient alteration, a more general case of the above expression (1) that is analogous to the OC case of Eq. (2) needs to be derived from the thermodynamic principles since the final configuration will consist of a PY material attached to a thermally active material, namely, NP material, which will then exert "thermally motivated external" stress onto the PY material under OC condition. So, we derive the expression for the PY coef. $\left(p_{i}\right)$ from its fundamental definition, ${ }^{1,9} p_{i}=\Delta P_{\mathrm{Si}} / \Delta \Theta=d P_{\mathrm{Si}} / d \Theta, i=1-3$, where $d P_{\mathrm{Si}}$ is the change in spontaneous polarization vector's component in the $i$-direction, $d \Theta$ is the change in temperature, $\Delta P_{\mathrm{Si}}$ is the dipole moment per unit volume, i.e., spontaneous polarization, in the $i$-direction, $\Delta \Theta$ is the uniform temperature change, and $p_{i}$ is the PY coef. in the $i$-direction. Also, D $=\varepsilon_{0} \mathbf{E}+\mathbf{P}$ in any dielectric material and $\mathbf{P}=\mathbf{P}_{S}+\mathbf{P}_{\text {ind }}$ in piezoor PY materials with $\mathbf{P}_{\text {ind }}=\varepsilon_{0} \boldsymbol{\chi}_{\mathrm{e}} \mathbf{E},{ }^{11}$ implying $\mathbf{D}=\varepsilon_{0} \boldsymbol{\varepsilon}_{r} \mathbf{E}$ $+\mathbf{P}_{S}$ and hence $P_{\mathrm{Si}}=D_{i}-\varepsilon_{0}\left(\varepsilon_{r}\right)_{i} E_{i}$. Therefore for OC condition $\left(d D_{i}=0\right)$,

$$
p_{i}=d P_{\mathrm{Si}} / d \Theta=-\varepsilon_{0}\left(\varepsilon_{r}\right)_{i} d E_{i} / d \Theta,
$$

where $\mathbf{P}$ is the total polarization, $\mathbf{P}_{S}$ is the spontaneous polarization, $\mathbf{D}$ is the electric displacement, $\mathbf{E}$ is the electric field (intensity), $\mathbf{P}_{\text {ind }}$ is the induced polarization owing to $\mathbf{E}$, $\boldsymbol{\chi}_{\mathrm{e}}=\boldsymbol{\varepsilon}_{\mathbf{r}}-\mathbf{I}$ is the dielectric susceptibility, $\mathbf{I}$ is the identity matrix/vector, $\varepsilon_{0}$ is the permittivity of free space, and $\boldsymbol{\varepsilon}_{r}$ is the relative dielectric constant.
Now, define Gibbs free energy, $G$, of a piezoelectric crystal as ${ }^{1,12} G=U-S_{i j} T_{k l}-D_{m} E_{n}-\sigma \Theta$, where $i, j, k, l, m, n$ $=1-3$. Also defining the temperature, stress, and electric displacement as the independent variables, we have $G=G\left(T_{i j}\right.$, $D_{m}$, and $\Theta$ ) and assuming constant electric displacement (i.e., $d D_{n}=0 \forall n$ ) for OC condition,

$$
\begin{aligned}
& d S_{i j}=\left(\partial S_{i j} / \partial T_{k l}\right)_{D, \Theta} d T_{k l}+\left(\partial S_{i j} / \partial \Theta\right)_{T, D} d \Theta, \\
& d E_{m}=\left(\partial E_{m} / \partial T_{k l}\right)_{D, \Theta} d T_{k l}+\left(\partial E_{m} / \partial \Theta\right)_{T, D} d \Theta .
\end{aligned}
$$

From Eq. (4) we get

$$
\begin{aligned}
\frac{d E_{m}}{d \Theta}= & \left(\partial E_{m} / \partial \Theta\right)_{T, D} \\
& -\left(\partial E_{m} / \partial T_{k l}\right)_{D, \Theta}\left(\partial S_{i j} / \partial T_{k l}\right)_{D, \Theta}{ }^{-1}\left[\left(\partial S_{i j} / \partial \Theta\right)_{T, D}\right. \\
& \left.-d S_{i j} / d \Theta\right]=\left(\frac{\partial E_{m}}{\partial D_{n}}\right)_{T, \Theta}\left(\frac{\partial D_{n}}{\partial \Theta}\right)_{T, D} \\
& -\left(\frac{\partial E_{m}}{\partial D_{n}}\right)_{T, \Theta}\left(\frac{\partial D_{n}}{\partial T_{k l}}\right)_{D, \Theta}\left(\partial S_{i j} / \partial T_{k l}\right)_{D, \Theta}{ }^{-1}\left[\left(\partial S_{i j} / \partial \Theta\right)_{T, D}\right. \\
& \left.-d S_{i j} / d \Theta\right]=\left(1 / \varepsilon_{0}\left(\varepsilon_{r}{ }^{T}\right)_{m}\right)\left[\left(\frac{\partial D_{n}}{\partial \Theta}\right)_{T, D}-\left(d_{m k l}^{D, \Theta}\right)\right. \\
& \left.\times\left(s_{i j k l}{ }^{D, \Theta}\right)^{-1}\left\{\alpha_{i j}^{T, D}-\frac{d S_{i j}}{d \Theta}\right\}\right],
\end{aligned}
$$

where $s_{i j k l}^{D, \Theta}$ is the elastic compliance at constant temperature and electric displacement.

Expressions (3) and (5) imply the following: Total PY coefficient under OC condition $=p_{m}$

$$
\begin{aligned}
= & -\varepsilon_{0}\left(\varepsilon_{r}^{T}\right)_{m} \frac{d E_{m}}{d \Theta}=-\varepsilon_{0}\left(\varepsilon_{r}^{T}\right)_{m}\left(1 / \varepsilon_{0}\left(\varepsilon_{r}^{T}\right)_{m}\right)\left[\left(\frac{\partial D_{n}}{\partial \Theta}\right)_{T, D}\right. \\
& \left.-\left(d_{m k l}^{D, \Theta}\right)\left(S_{i j k l}^{D, \Theta}\right)^{-1}\left\{\alpha_{i j}^{T, D}-\frac{d S_{i j}}{d \Theta}\right\}\right]=-\left(\frac{\partial D_{n}}{\partial \Theta}\right)_{T, D} \\
& +\left(d_{m k l}^{D, \Theta}\right)\left(S_{i j k l}^{D, \Theta}\right)^{-1}\left\{\alpha_{i j}^{T, D}-\frac{d S_{i j}}{d \Theta}\right\}=P_{m}^{T, D} \\
& +\left(d_{m k l}{ }^{D, \Theta}\right)\left(S_{i j k l}^{D, \Theta}\right)^{-1}\left\{\alpha_{i j}^{T, D}-\frac{d S_{i j}}{d \Theta}\right\}=p_{m}^{T, D} \\
& +\left(d_{m k l}{ }^{D, \Theta}\right)\left(c_{i j k l}{ }^{D, \Theta}\right)\left\{\alpha_{i j}^{T, D}-\frac{d S_{i j}}{d \Theta}\right\} .
\end{aligned}
$$

Notice the change in the sign of the secondary PY coefficient from that of SC condition (2). This indicates that the PY coefficient under OC can vary greatly from that under SC condition since where the secondary effect is an enhancement will lead to reduction and vice versa for these two differing conditions, provided the same amount of strain is applied. Indeed, our analysis illustrates that the enhancement under SC leads to reduction under OC, but due to the sheer magnitude of the secondary effect it can lead to change in sign in the PY coefficient itself where very large alteration of the PY coefficient is achieved at very low thickness ratios $R$ for PY materials that demonstrated relatively large enhancements under SC such as PZT and barium titanate (BTO). 
It is evident from Eq. (6) that larger the strain the nonpyroelectric (NP) component can exert on PY component and greater the piezoelectric coefficient of the PY material, bigger the change in secondary contribution. This leads to the conclusion that stiffer NP material with greater disparity in thermal expansion coefficient $(\alpha)$ with that of PY and more compliant PY material with high piezoelectric coefficients would lead to largest PY coefficient alteration.

\section{B. Material properties under $\mathrm{OC}$ condition}

Before we move on to evaluating the PY coefficients under OC condition, we must first mention some of the relationship between various material properties evaluated under SC and OC. The information on the material properties required for the evaluation of Eq. (2) is largely available from various sources such as the manufacturers. ${ }^{13-15}$ However, Eq. (6) poses significantly more difficult challenge since most manufacturers provide measurements evaluated under SC. Therefore, here we establish some of the relations between the two sets of parameters based on various sources. ${ }^{1,12,16,17}$

\section{Primary PY coefficients under SC and OC}

There is little in the literature which explicitly deals with this issue. As the primary PY coefficient is an intrinsic property of the material, we assume the following equality relationship between the primary PY coefficients under SC and OC, namely, $P_{m}{ }^{S, E}$ and $P_{m}{ }^{S, D}$, respectively; $p_{m}{ }^{S, E}=p_{m}{ }^{S, D}$. From Eqs. (1), (2), and (6) we have

$$
\begin{aligned}
p_{m}{ }^{T, E} & -\left(d_{m k l}{ }^{E, \Theta}\right)\left(c_{i j k l}{ }^{E, \Theta}\right)\left(\alpha_{i j}{ }^{T, E}\right)=p_{m}{ }^{T, D}+\left(d_{m k l}{ }^{D, \Theta}\right) \\
& \times\left(c_{i j k l}{ }^{D, \Theta}\right)\left(\alpha_{i j}^{T, D}\right),
\end{aligned}
$$

which implies

$$
\begin{aligned}
p_{m}{ }^{T, D}= & p_{m}{ }^{T, E}-\left(d_{m k l}{ }^{E, \Theta}\right)\left(c_{i j k l}{ }^{E, \Theta}\right)\left(\alpha_{i j}{ }^{T, E}\right)-\left(d_{m k l}{ }^{D, \Theta}\right) \\
& \times\left(c_{i j k l}{ }^{D, \Theta}\right)\left(\alpha_{i j}{ }^{T, D}\right) .
\end{aligned}
$$

We notice the fact that there is no distinction between the primary coefficients under SC and OC in the work of Grout et $a .^{18}$ and the same value for the PY coefficient was used for both SC and OC cases of the PY expression derived by Ploss et al. ${ }^{19}$ which supports this equality assumption.

\section{Piezoelectric constants under SC and OC}

Conventionally, the piezoelectric constants are assumed to be the same under both SC and OC since the elastic coefficients, which will be multiplied to the piezoelectric constant to describe the overall piezoelectric effect, will reflect the consequences of this electrical condition on the overall piezoelectric effect. Hence, $d_{m k l}^{E, \Theta}=d_{m k l}{ }^{D, \Theta}$.

\section{Elastic compliances under SC and OC (References 1, 12, and 20)}

This is well known in literature as

$$
s_{i j k l}^{D, \Theta}-s_{i j k l}^{E, \Theta}=-\left(d_{m i j}{ }^{E, \Theta}\right)\left(d_{n k l}^{E, \Theta}\right)\left(\beta_{m n}{ }^{T, \Theta}\right),
$$

and

$$
c_{i j k l}^{D, \Theta}-c_{i j k l}^{E, \Theta}=\left(e_{i j m}{ }^{\Theta}\right)\left(e_{k l w}{ }^{\Theta}\right)\left(\beta_{m w}{ }^{S, \Theta}\right),
$$

where $\beta_{m n}{ }^{T, \Theta}$ and $\beta_{m n}{ }^{S, \Theta}$ are components from the inverse of the permittivity tensor and $e_{i j m}{ }^{\Theta}$ is the piezoelectric constant (stress/electric field) equaling to $c_{i j k l}^{E, \Theta} d_{k l m}^{E, \Theta}$.

\section{Thermal expansion coefficients under SC and OC (References 1 and 20)}

Nye's book ${ }^{1}$ contains the following relationship for the thermal expansion coefficients:

$$
\alpha_{i j}^{T, D}-\alpha_{i j}^{T, E}=-\left(d_{k i j}^{E, \Theta}\right)\left(\beta_{k l}^{T, \Theta}\right) p_{l}^{T, E} .
$$

Using the previously stated four relations between the parameters under SC and OC, it is possible to evaluate Eq. (6) in terms of the parameters used in Eq. (2). For the purpose of this article, following expression for the PY coefficient under OC will be used as the general form, but it should also be noted that other forms are also easily derivable from the four relations. From Eq. (6) and the four relations,

$$
\begin{aligned}
p_{m}{ }^{\mathrm{OC}}= & p_{m}{ }^{T, D}+\left(d_{m k l}{ }^{D, \Theta}\right)\left(c_{i j k l}{ }^{D, \Theta}\right)\left\{\alpha_{i j}{ }^{T, D}-\frac{d S_{i j}}{d \Theta}\right\}=p_{m}{ }^{T, E} \\
& -\left(d_{m k l}{ }^{E, \Theta}\right)\left(c_{i j k l}{ }^{E, \Theta}\right)\left(\alpha_{i j}{ }^{T, E}\right)-\left(d_{m k l}{ }^{D, \Theta}\right)\left(c_{i j k l}{ }^{D, \Theta}\right) \\
& \times\left(\alpha_{i j}{ }^{T, D}\right)+\left(d_{m k l}{ }^{D, \Theta}\right)\left(c_{i j k l}{ }^{D, \Theta}\right)\left\{\alpha_{i j}{ }^{T, D}-\frac{d S_{i j}}{d \Theta}\right\} \\
= & p_{m}{ }^{T, E}-\left(d_{m k l}{ }^{E, \Theta}\right)\left(c_{i j k l}{ }^{E, \Theta}\right)\left(\alpha_{i j}{ }^{T, E}\right)-\left(d_{m k l}{ }^{D, \Theta}\right) \\
& \times\left(c_{i j k l}{ }^{D, \Theta}\right)\left(\frac{d S_{i j}}{d \Theta}\right), \quad \text { where } i, j, k, l, m=1-3 \quad
\end{aligned}
$$

\section{Force balance equation and its solution}

The same solution as the one presented in our other communication $^{3}$ will be used in our analysis although all the material properties of the PY material will be replaced with their OC counterparts, $c_{i j}=c_{i j}{ }^{D, \Theta}$ not $c_{i j}{ }^{E, \Theta}$, for example. Plate theory ${ }^{21}$ and force balance equations were used to elicit the strain NP can exert on PY. As the system is symmetrical about the 1-2 plane, two layer plate theory should present a good approximation to our three layer case. From the generalized Hooke's law for orthotropic materials ${ }^{21}$ with the assumptions of the Kirchhoff plate conditions, ${ }^{22}$ i.e., only $S_{1}$, $S_{2}, S_{3}$, and $S_{6}$ are nonzero (but $T_{3}=T_{4}=T_{5}=0$ ), and that the shear stress in the 1-2 plane, i.e., $T_{6}=\tau_{12}$ is negligible, the total strain experienced by PY can be derived to be

$$
d{ }^{\mathrm{PY}} S_{1}=d{ }^{\mathrm{PY}} S_{2}=\frac{Y\left(1-2 s_{13} c_{13}\right)\left(1+{ }^{\mathrm{PY}} \alpha d \Theta\right)\left({ }^{\mathrm{NP}} \alpha-{ }^{\mathrm{PY}} \alpha\right) d \Theta}{(1-\nu)\left(c_{11}+c_{12}\right)\left(1+{ }^{\mathrm{NP}} \alpha d \Theta\right) R+Y\left(1-2 s_{13} c_{13}\right)\left(1+{ }^{\mathrm{PY}} \alpha d \Theta\right)}+{ }^{\mathrm{PY}} \alpha d \Theta
$$


and

$$
d{ }^{\mathrm{PY}} S_{3}=\frac{2 Y s_{13}\left(c_{11}+c_{12}\right)\left(1+{ }^{\mathrm{PY}} \alpha d \Theta\right)\left({ }^{\mathrm{NP}} \alpha-{ }^{\mathrm{PY}} \alpha\right) d \Theta}{(1-\nu)\left(c_{11}+c_{12}\right)\left(1+{ }^{\mathrm{NP}} \alpha d \Theta\right) R+Y\left(1-2 s_{13} c_{13}\right)\left(1+{ }^{\mathrm{PY}} \alpha d \Theta\right)}+{ }^{\mathrm{PY}} \alpha d \Theta,
$$

where $Y$ and $\nu$ are Young's modulus and Poisson's ratio of $\mathrm{NP}$, respectively, $s_{i j}$ is the elastic compliance of PY, $c_{i j}$ is the elastic stiffness of PY, and $R$ is the thickness ratio of $\mathrm{PY}$ to NP layers.

Substituting Eq. (8) into Eq. (7) yields the PY coefficient of our structures, depicting the magnitude of our alteration. It must be noted that although Eq. (7) is applicable to any PY material universally, Eq. (8) is a simplified form of the solution to the force balance equation with certain assumptions on the symmetry of the PY material assumed, which all but one, namely, polyvinylidene fluoride (PVDF), PY materials explored in this article suffice. The assumptions made are ${ }^{\mathrm{PY}} \alpha={ }^{\mathrm{PY}} \alpha_{j}$ for all $j=1-2, s_{13}=s_{23}, c_{11}=c_{22}, c_{12}=c_{21}$, and $c_{23}=c_{31}$. PVDF does not satisfy these relations, and hence for PVDF we implemented the full solution to the force balance equation, the consequences of which are elucidated in Sec. III E, although the solution itself is not presented in this treatise due to space constraints.

\section{Thermal budget and efficiency}

We define a measure termed "efficiency (eff)" and use it to measure how efficiently our PY structures convert heat energy into electricity, and compare that of PY material alone and our altered laminate composite.

In order to make comparison, we also define a "figure of merit for efficiency" by $F_{\text {eff }}={ }^{\text {com }}$ eff $/{ }^{P Y}$ eff, where ${ }^{P Y}$ eff denotes the efficiency of the pure PY material and ${ }^{\text {com }}$ eff that of composite. Depending on the application, we have two different expressions for $F_{\text {eff }}$.

(a) First is the ratio between the same total volume of PY material and 2-2 connectivity composite, namely, $F^{a}$ eff, which will result in the ratio between a 2-2 connectiv- ity composite and a PY material with the same thickness as the total thickness of the composite (this means the thickness of the PY material used in the composite is thinner than the stand alone PY material). Assume the total volume for both cases to be $t_{3} L W$,

$$
\begin{aligned}
F_{\text {eff }}^{a}= & \frac{{ }_{\text {eff }}^{\mathrm{PY}} \text { eff }}{{ }^{\mathrm{com}} p_{3}} \frac{\frac{t_{3} L W}{R+1}\left[{ }^{\mathrm{PY}} c_{\mathrm{vol}} R+{ }^{\mathrm{NP}} c_{\mathrm{vol}}\right]}{\frac{{ }^{\mathrm{PY}} p_{3}}{t_{3} L W^{\mathrm{PY}} c_{\mathrm{vol}}}} \\
= & \frac{{ }^{\mathrm{com}} p_{3}{ }^{\mathrm{PY}} c_{\mathrm{vol}}[R+1]}{{ }_{p_{3}}\left[{ }^{\mathrm{PY}} c_{\mathrm{vol}} R+{ }^{\mathrm{NP}} c_{\mathrm{vol}}\right]},
\end{aligned}
$$

where ${ }^{\mathrm{PY}} p_{3}$ is PY coef. of PY material, ${ }^{\mathrm{com}} p_{3}$ PY coef. of composite, $L$ is the length, $W$ is the width, $t_{3}$ is the total thickness $\left(={ }^{\mathrm{PY}} t+{ }^{\mathrm{NP}} t\right),{ }^{\mathrm{PY}} t$ is the thickness of $\mathrm{PY},{ }^{\mathrm{NP}} t$ is the thickness of NP, $R$ is the thickness ratio $\left(={ }^{\mathrm{PY}} t /{ }^{\mathrm{NP}} t\right)$, and ${ }^{\mathrm{PY}} c_{\mathrm{vol}}$ or ${ }^{\mathrm{NP}} c_{\mathrm{vol}}$ is the volumetric heat capacity of PY or NP. If $F_{\text {eff }}^{a}>1$, then this denotes an improvement in the thermal-to-electrical conversion efficiency compared with that of pure PY material, while $F_{\text {eff }}^{a}<1$ implies an inferior conversion performance. Since both the composite and the PY material are of the same volume, this ratio will indicate an improvement as long as ${ }^{\mathrm{PY}} c_{\mathrm{vol}}>{ }^{\mathrm{NP}} c_{\mathrm{vol}}$ and ${ }^{\mathrm{com}} p_{3}>{ }^{\mathrm{PY}} p_{3}$.

(b) Another ratio is between a PY material and a composite with the PY material of the same thickness,

TABLE I. Thermal coefficients of various PY materials under short and OC conditions. [Units: $\alpha^{E}$ and $\alpha^{D}$ $\left(\times 10^{-6} \mathrm{~m} \mathrm{~m}^{-1} \mathrm{~K}^{-1}\right) ; c_{\mathrm{vol}}\left(\times 10^{6} \mathrm{~J} \mathrm{~m}^{-3} \mathrm{~K}^{-1}\right)$.]

\begin{tabular}{lcccccc}
\hline \hline & PZT-5H & PZT-5A & BTO $^{\mathrm{a}}$ & LTO $^{\mathrm{b}}$ & LNO $^{\mathrm{b}}$ & PVDF $^{2}$ \\
\hline$\alpha_{1}{ }^{E}$ & $3.0^{\mathrm{c}}$ & $4.0^{\mathrm{c}}$ & 15.7 & 16 & 15 & $13^{\mathrm{d}}$ \\
$\alpha_{2}{ }^{E}$ & $3.0^{\mathrm{c}}$ & $4.0^{\mathrm{c}}$ & 15.7 & 16 & 15 & $145^{\mathrm{d}}$ \\
$\alpha_{3}{ }^{E}$ & $3.0^{\mathrm{c}}$ & $4.0^{\mathrm{c}}$ & 6.2 & 4 & 7.5 & $80^{\mathrm{e}}$ \\
$\alpha_{1}{ }^{D}$ & -3.3 & 0.4 & 11.1 & 14.8 & 14.7 & 21.5 \\
$\alpha_{2}{ }^{D}$ & -3.3 & 0.4 & 11.1 & 14.8 & 14.7 & 145.9 \\
$\alpha_{3}{ }^{D}$ & 15.8 & 11.3 & 17.7 & 8.6 & 9.4 & 67.4 \\
$c_{\text {vol }}$ & $3.15^{\mathrm{f}}$ & $3.15^{\mathrm{f}}$ & 3.19 & 1.87 & 2.92 & $2.3^{\mathrm{g}}$ \\
\hline \hline
\end{tabular}

${ }^{\mathrm{a}}$ Reference 39.

${ }^{\mathrm{b}}$ Reference 40.

${ }^{\mathrm{c}}$ Reference 14.

${ }^{\mathrm{d}}$ References 27 and 28.

${ }^{\mathrm{e}}$ Reference 41.

${ }^{\mathrm{f}}$ References 15 and 42 .

${ }^{\mathrm{g}}$ Reference 16. 
TABLE II. PY coefficients of various PY materials under OC condition. [Units: $P_{3}^{T, E}$ and $P_{3}^{T, D}$; $\left(\times 10^{-4} \mathrm{C} \mathrm{m}^{-2} \mathrm{~K}^{-1}\right)$.]

\begin{tabular}{lllllll}
\hline \hline & PZT-5H $^{\mathrm{a}}$ & $\mathrm{PZT}^{-5 A^{\mathrm{a}}}$ & \multicolumn{1}{c}{$\mathrm{BTO}^{\mathrm{b}}$} & \multicolumn{1}{c}{ LTO $^{\mathrm{c}}$} & LNO $^{\mathrm{c}}$ & PVDF $^{\mathrm{d}}$ \\
\hline$P_{3}{ }^{T, E}$ & -5.0 & -3.0 & -2.0 & -2.3 & -0.83 & -0.274 \\
$P_{3}{ }^{T, D}$ & -23.120 & -4.078 & -1.461 & -2.598 & -1.173 & -0.261 \\
\hline \hline
\end{tabular}

${ }^{\mathrm{a}}$ References 14 and 42 .

${ }^{\mathrm{b}}$ Refrences 23 and 24 .

${ }^{c}$ Reference 40.

${ }^{\mathrm{d}}$ References 27 and 30 .

$$
\begin{aligned}
& F_{\text {eff }}^{b}=\frac{{ }_{\mathrm{com}}^{\mathrm{eff}}}{{ }_{\mathrm{eff}}}=\frac{\frac{{ }^{\mathrm{com}} p_{3}}{R+1}\left[{ }^{\mathrm{PY}} c_{\mathrm{vol}} R+{ }^{\mathrm{NP}} c_{\mathrm{vol}}\right]}{\frac{{ }^{\mathrm{PY}} p_{3}}{\mathrm{PY}_{t} L W^{\mathrm{PY}} c_{\mathrm{vol}}}} \\
& =\frac{\frac{t_{3} L W}{R+1}\left[{ }^{\mathrm{PY}} c_{\mathrm{vol}} R+{ }^{\mathrm{NP}} c_{\mathrm{vol}}\right]}{\frac{R+1}{t_{3} R} \frac{{ }^{\mathrm{PY}} p_{3}}{L W^{\mathrm{PY}} c_{\mathrm{vol}}}}=\frac{{ }^{\mathrm{com}} p_{3}{ }^{\mathrm{PY}} c_{\mathrm{vol}} R}{p_{3}\left[{ }^{\mathrm{PY}} c_{\mathrm{vol}} R+{ }^{\mathrm{NP}} c_{\mathrm{vol}}\right]} .
\end{aligned}
$$

Once more, $F_{\text {eff }}^{b}>1$ signifies increased efficiency.

\section{RESULTS AND DISCUSSION}

A few of the most widely used PY materials, such as PZT (PZT-5H and PZT-5A), ${ }^{14,15}$ BTO, ${ }^{23-25}$ lithium tantalate (LTO), ${ }^{26}$ lithium niobate (LNO), ${ }^{26}$ and $\mathrm{PVDF}^{27-30}$ were paired with six different NP materials with wide ranging thermal and elastic properties, namely, stainless steel (St), ${ }^{13}$ polytetrafluoroethylene (PTFE or Teflon), ${ }^{31}$ chlorinated polyvinyl chloride (CPVC) thermoplastic, ${ }^{32-34}$ aluminum, ${ }^{34,35}$ zinc, ${ }^{34,36}$ and Invar $36,{ }^{37,38}$ to analyze their OC PY coefficient alteration credentials. Although all the 36 pairs were examined, in this communication the conclusions of only selected few with the most interesting results are presented.

Tables I and II elucidate the difference between material properties under SC and OC. In particular, materials with high $p_{m}{ }^{T, E}$ such as PZT, BTO, and LTO show the largest change in their thermal expansion coefficient due to the fourth relation stated earlier in Sec. II B. This significant change in their thermal expansion behavior also leads to substantial change in their secondary contribution to the overall PY coefficient under OC, $p_{m}{ }^{T, D}$. For PZT, LTO, and LNO the magnitude of PY coefficient is greater under OC, while the opposite is true for others as illustrated in Table II. This suggests that where secondary PY coefficient is concerned, it is rather difficult to anticipate its contribution to overall PY coefficient under both SC and OC condition until all the components, such as the thermal expansion coefficients and signs of the secondary part, are all identified and their interactions assessed for the overall impact. Table III further reinforces this view as the signs of the secondary PY coefficients are only different from that of under SC in materials such as LTO, LNO, and PVDF despite the expected change of sign from Eq. (6), owing largely to significant change in the thermal expansion coefficients as displayed in Table I. For instance, although Eq. (6) suggests PZT-5H's secondary PY coefficient under OC could have the opposite sign from that under SC, valued at $-0.473 \times 10^{-4} \mathrm{C} \mathrm{m}^{-2} \mathrm{~K}^{-1}$, it actually evaluates to $-18.593 \times 10^{-4} \mathrm{C} \mathrm{m}^{-2} \mathrm{~K}^{-1}$ since its thermal expansion coefficients are altered so drastically under OC as apparent from Table I. Meanwhile, LTO's secondary PY coef. under OC valued at $-0.195 \times 10^{-4} \mathrm{C} \mathrm{m}^{-2} \mathrm{~K}^{-1}$ has the opposite sign from that under $\mathrm{SC}, 0.103 \times 10^{-4} \mathrm{C} \mathrm{m}^{-2} \mathrm{~K}^{-1}$ as the thermal expansion coefficients of LTO are not changed as severely from the transition of electrical conditions.

\section{A. PZT-5H}

Since the behavior of PZT-5A is very similar to that of PZT-5H, only the results of PZT-5H will be provided. It is evident from Tables I and II that PZT-5H is the main beneficiary of the increased PY coefficient under OC owing to

\begin{tabular}{|c|c|c|c|c|c|c|}
\hline & PZT-5H & PZT-5A & BTO & LTO & LNO & PVDF \\
\hline Young's modulus $\left(\times 10^{9} \mathrm{~N} \mathrm{~m}^{-2}\right)$ & 185.6 & 162.0 & 282.4 & 240.0 & 219.9 & 3.6 \\
\hline $\operatorname{dc} 1\left(\mathrm{C} \mathrm{m}^{-2}\right)$ & -82.749 & -21.662 & -4.045 & 0.091 & 0.200 & 0.0362 \\
\hline $\mathrm{dc} 2\left(\mathrm{C} \mathrm{m}^{-2}\right)$ & -82.749 & -21.662 & -4.045 & 0.091 & 0.200 & 0.0004 \\
\hline $\mathrm{dc} 3\left(\mathrm{C} \mathrm{m}^{-2}\right)$ & 83.390 & 16.172 & 5.497 & 1.948 & 1.359 & -0.0179 \\
\hline Primary PY coef. $\left(\times 10^{-4} \mathrm{C} \mathrm{m}^{-2} \mathrm{~K}^{-1}\right)$ & -4.527 & -2.432 & -1.382 & -2.403 & -0.987 & -0.265 \\
\hline SC secondary PY coef. $\left(\times 10^{-4} \mathrm{C} \mathrm{m}^{-2} \mathrm{~K}^{-1}\right)$ & -0.473 & -0.568 & -0.618 & 0.103 & 0.152 & -0.009 \\
\hline OC Secondary PY coef. $\left(\times 10^{-4} \mathrm{C} \mathrm{m}^{-2} \mathrm{~K}^{-1}\right)$ & -18.593 & -1.646 & -0.079 & -0.195 & -0.186 & 0.004 \\
\hline PY coef. before enhancement $\left(\times 10^{-4} \mathrm{C} \mathrm{m}^{-2} \mathrm{~K}^{-1}\right)$ & -23.120 & -4.078 & -1.461 & -2.598 & -1.173 & -0.261 \\
\hline $\begin{array}{l}\text { Largest PY coef. after enhancement at } R=0.2( \\
\left.\times 10^{-4} \mathrm{C} \mathrm{m}^{-2} \mathrm{~K}^{-1}\right)\end{array}$ & 22.648 & 11.551 & -2.801 & -2.700 & -1.174 & -0.270 \\
\hline
\end{tabular}

TABLE III. PY materials assessment for OC condition. 


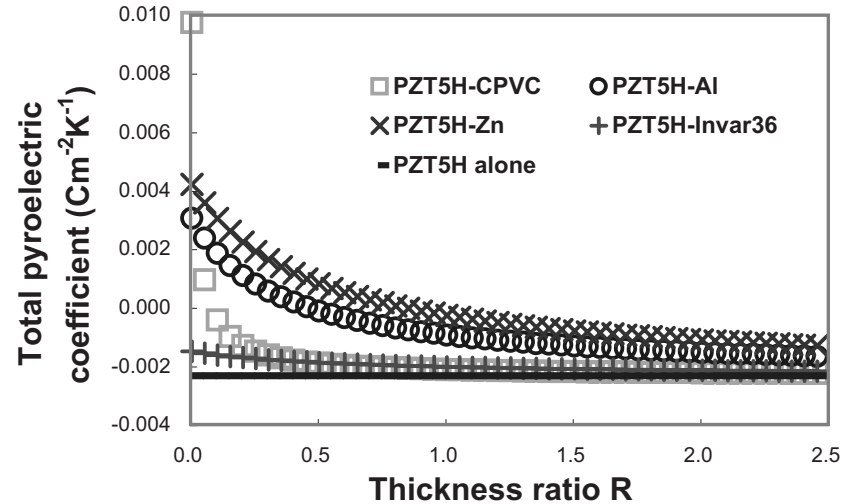

FIG. 1. Total PY coefficient vs thickness ratio PY to NP for PZT-5H pairs under $\mathrm{OC}$.

substantial changes in its thermal expansion coefficients as consequences of high PY coefficient under SC. Its PY coefficient under $\mathrm{OC}$ is $-23.1 \times 10^{-4} \mathrm{C} \mathrm{m}^{-2} \mathrm{~K}^{-1}$, whereas under SC this is only $-5.0 \times 10^{-4} \mathrm{C} \mathrm{m}^{-2} \mathrm{~K}^{-1}$. In addition, its $\mathrm{dc} 1=\left(\mathrm{d}_{3 \mathrm{i}}\right)\left(\mathrm{c}_{1 \mathrm{i}}\right), \mathrm{dc} 2=\left(\mathrm{d}_{3 \mathrm{i}}\right)\left(\mathrm{c}_{2 \mathrm{i}}\right)$, and $\mathrm{dc} 3=\left(\mathrm{d}_{3 \mathrm{i}}\right)\left(\mathrm{c}_{3 \mathrm{i}}\right)$ values under $\mathrm{OC}$ is also about 5.4 times larger than that under $\mathrm{SC}$, leading to larger contribution from the secondary effect to the overall PY coefficient, as depicted in Fig. 1. It is evident from Fig. 1 that what was an enhancement under SC at low thickness ratios $(R)$ is a reduction under OC due to the sign of the secondary effect demonstrated in Eq. (6), meanwhile the secondary contribution can be so large at very low $R$ values that it actually switches the sign of the overall PY coefficient to the positive region peaking at huge PY coefficient of $97 \times 10^{-4} \mathrm{C} \mathrm{m}^{-2} \mathrm{~K}^{-1}$ at $R=0.005$ (minimum $R$ value evaluated, namely, $\left.R_{\min }\right)$ for PZT-5H-CPVC thermoplastic pair and around $40 \times 10^{-4} \mathrm{C} \mathrm{m}^{-2} \mathrm{~K}^{-1}$ at $R=R_{\min }$ for PZT$5 \mathrm{H}$-Zinc ( $\mathrm{Zn})$ pair. The $R$ value at which the sign change in the total PY coefficient occurs is smaller for NP materials with lower Young's modulus and thermal expansion coefficients as expected.

Figure 2 describes the figure of merit for efficiency belonging to the same volume of PZT-5H and its composites $\left(F_{\text {eff }}^{a}\right)$. They all reach the value of zero at certain values of $R$ owing to the PY coefficient switching between negative and positive signs at low $R$ values. PZT-5H-CPVC pair peaks to around 9.4 at $R_{\min }(R=0.005)$ while PZT-5H-PTFE reaches its maximum of approximately 7.0 at the same $R_{\min }$. It is apparent from Fig. 2 that both pairs mentioned above have

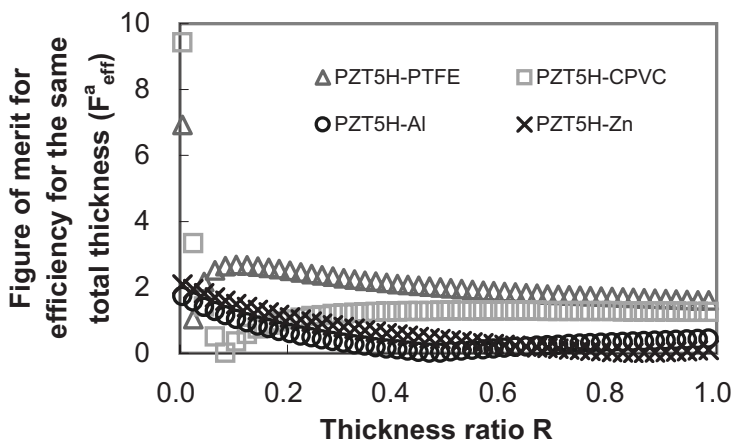

FIG. 2. Figure of merit for efficiency belonging to the same volume of PZT-5H composites vs thickness ratio PY to NP under OC.

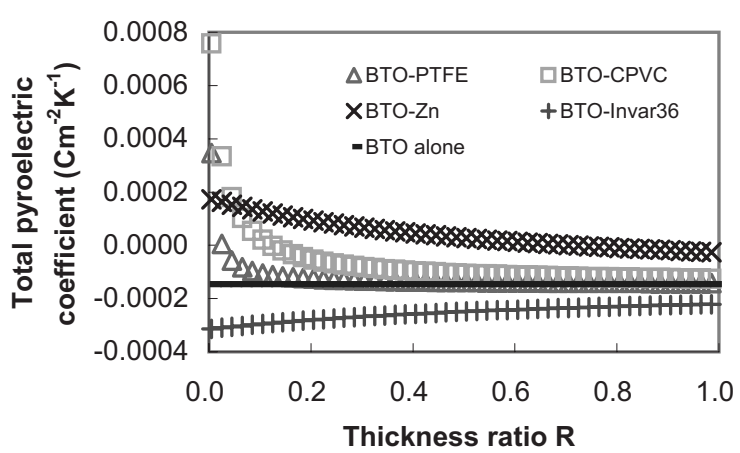

FIG. 3. Total PY coefficient vs thickness ratio PY to NP for BTO pairs under OC.

reasonably high $F_{\text {eff }}^{a}$, between 2.6 and 1.3 , for the whole of the $R$ range considered, insinuating improvement in the efficiencies of PZT-5H when CPVC or PTFE is attached. Zn and Al pairs fail to reach figure of merit for efficiency of higher than one except at $R<0.230$ and $R<0.110$, respectively, and this trend is expected to continue at $R$ values higher than one as increasing $R$ means less volume of NP material attached and hence the overall PY coefficient converging to that of PZT-5H alone.

\section{B. BTO}

BTO and PVDF were the only PY materials that experienced reduced PY coefficient under OC when compared to that evaluated under SC. Although BTO's thermal expansion coefficients also experienced quite a large change, $\alpha_{3}{ }^{D}$ in particular as exhibited in Table I, the change in its dc1, dc2, and dc 3 meant that the overall secondary effect under OC is drastically reduced to approximately -0.079 $\times 10^{-4} \mathrm{C} \mathrm{m}^{-2} \mathrm{~K}^{-1}$, which is only about one eighth of that under SC.

As is evident from Fig. 3, BTO-CPVC attains the highest total PY coefficient of $7.6 \times 10^{-4} \mathrm{C} \mathrm{m}^{-2} \mathrm{~K}^{-1}$ at $R=R_{\min }$ and maintains its superiority until $R=0.05$ at which point BTO-Zn takes over as the dominant pair. However, BTOInvar36 is the only pair that consistently outperforms BTO's own PY coefficient as it is the only pair that has PY coefficient more negative than $-1.461 \times 10^{-4} \mathrm{C} \mathrm{m}^{-2} \mathrm{~K}^{-1}$, the PY coefficient of BTO under OC, with maximum value of approximately $-3.14 \times 10^{-4} \mathrm{C} \mathrm{m}^{-2} \mathrm{~K}^{-1}$ at $R_{\min }$, owing to Invar36's unusually small thermal expansion coefficient. Fig-

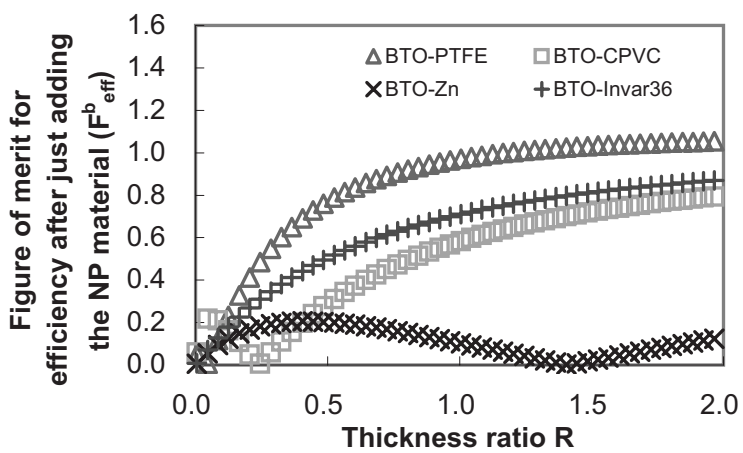

FIG. 4. Figure of merit for efficiency when NP material is added to BTO vs thickness ratio PY to NP under OC. 


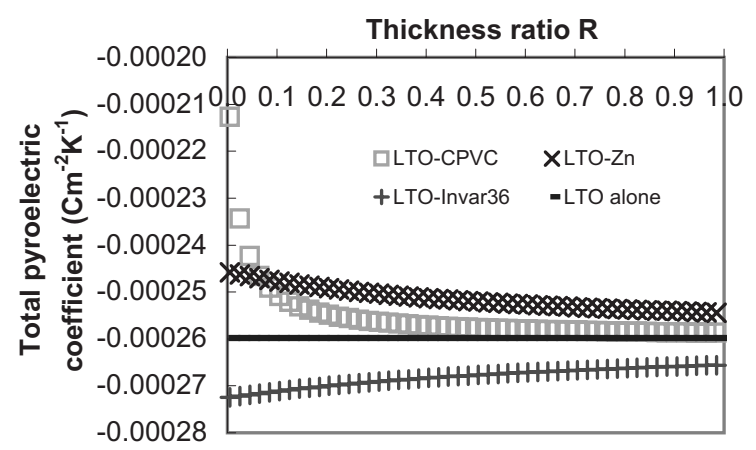

FIG. 5. Total PY coefficient vs thickness ratio PY to NP for LTO pairs under OC.

ure of merit for efficiency when NP materials are added to BTO, leading to increased volume, is presented in Fig. 4. Although some enhancement on the magnitude of the PY coefficient is achieved, it is not enough to drive $F_{\text {eff }}^{b}$ to higher than one in all the pairs except one. BTO-PTFE pair is the only one which possesses $F_{\text {eff }}^{b}>1$ reaching maximum of 1.05 at $R=2.00$, while all the others indicate reduction in efficiency due to increased thermal mass. BTO-Invar36 is the second best performing pair for BTO, which is quite surprising considering Invar36's rather high volumetric heat capacity of $5.15 \times 10^{6} \mathrm{~J} \mathrm{~m}^{-3} \mathrm{~K}^{-1}$, the highest among all $\mathrm{NP}$ and PY materials considered.

\section{LTO}

Figure 5 displays the PY coefficients for LTO pairs. All the pairs except LTO-Invar36 show reduction in the PY coefficient with LTO-CPVC reaching minimum of -2.127 $\times 10^{-4} \mathrm{C} \mathrm{m}^{-2} \mathrm{~K}^{-1}$ and LTO-Invar36 maximum of -2.725 $\times 10^{-4} \mathrm{C} \mathrm{m}^{-2} \mathrm{~K}^{-1}$ at $R_{\min }$. All in all, LTO behaves similar to BTO with the exception of sign change in the PY coefficients owing to the limited secondary effect contribution to the overall PY coefficient. Although most of the pairs show reduction in the PY coefficient magnitude, some manage to show improvement in efficiency where the same volume of the composites are concerned $\left(F_{\text {eff }}^{a}\right)$ as illustrated in Fig. 6. LTO-PTFE pair exhibits peak of approximately 2.4 at $R$ $=0.025$ and maintains $F_{\text {eff }}^{a}>1$ throughout the $R$ range investigated with the minimum of 1.4 at $R=1.005$. LTO-CPVC also manages $F_{\text {eff }}^{a}>1$ throughout the $R$ range although at

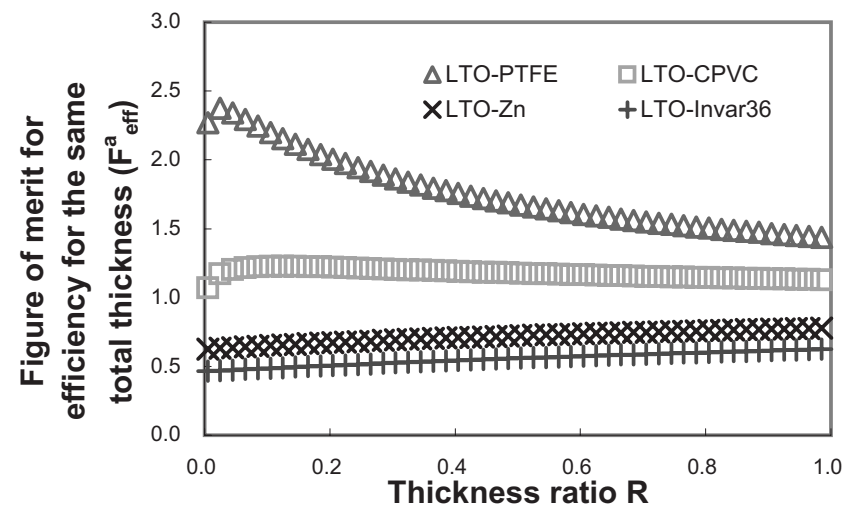

FIG. 6. Figure of merit for efficiency belonging to the same volume of LTO composites vs thickness ratio PY to NP under OC.

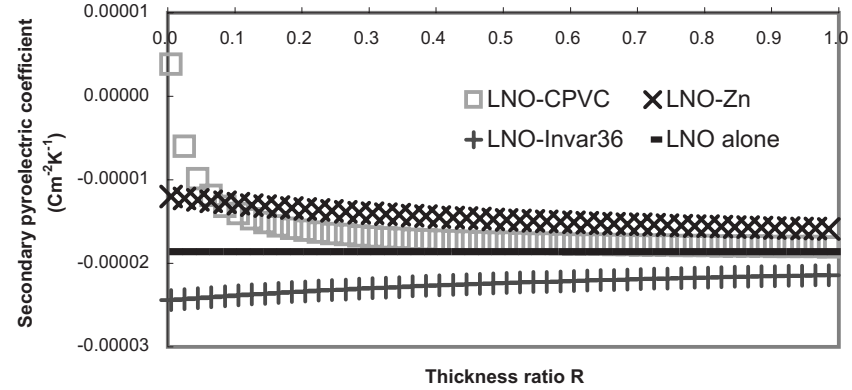

FIG. 7. Secondary PY contribution from NP vs thickness ratio PY to NP for LNO pairs under OC.

much smaller values of $1.1<F_{\text {eff }}^{a}<1.2$. The reasons behind these improvements are the exceptionally low volumetric heat capacities of PTFE and CPVC, again leading to good $F_{\text {eff }}^{a}$ values.

\section{LNO}

The behaviors of the PY coefficients of LNO pairs are very similar to that of LTO pairs in Fig. 5. Figure 7 demonstrates how similar they are by displaying the secondary PY effect contributions to the overall PY coefficient arising from the introduction of the NP materials. LNO-Invar36 is again the only pair that exhibits enhancement in the secondary PY coefficient, and hence the overall PY coefficient, while LNO-Zn shows reduction but falls short of changing the sign of the secondary effect. LNO-CPVC, however, displays both reduction and change in sign in the secondary PY coefficient. The maximum secondary PY coefficient observed is $-0.244 \times 10^{-4} \mathrm{C} \mathrm{m}^{-2} \mathrm{~K}^{-1}$ at $R_{\min }$ by LNO-Invar36 pair, which corresponds to around $31 \%$ increase in the secondary PY coefficient from the introduction of Invar36 to LNO. As evident from Fig. 8, where $F^{a}$ eff is concerned LNO-PTFE performs the best with the optimum $F_{\text {eff }}^{a}$ value of 3.63 at $R=0.025$ due to PTFE's lowest volumetric heat capacity among all the materials investigated. LNO-CPVC pair does not fare too badly either with the optimum of 1.84 at $R$ $=0.065$, while other pairs exhibiting improvement in the efficiency also include LNO-Al and LNO-Zn, whose $F^{a}$ eff lie very near to one.

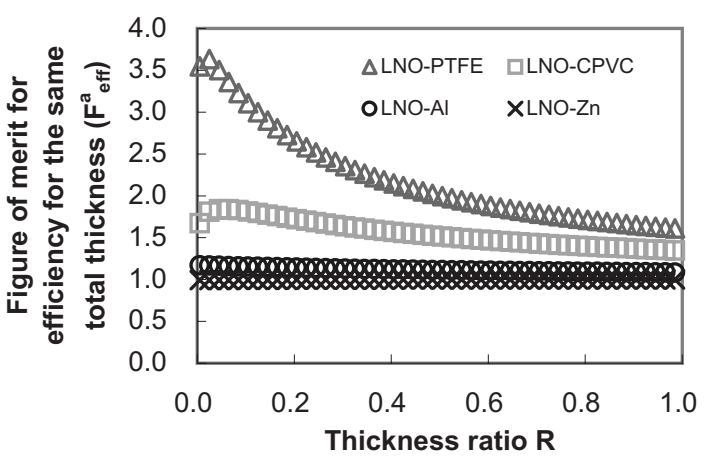

FIG. 8. Figure of merit for efficiency belonging to the same volume of LNO composites vs thickness ratio PY to NP under OC. 


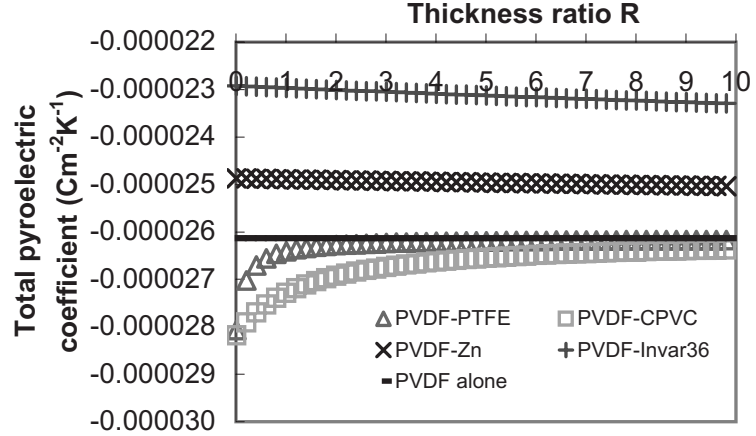

FIG. 9. Total PY coefficient vs thickness ratio PY to NP for PVDF pairs under OC.

\section{E. PVDF}

As mentioned earlier, PVDF experienced reduced PY coefficient under OC. Although the difference in the magnitude of the overall PY coefficient is quite small, the introduction of NP materials does affect the PY coefficient of PVDF as depicted in Fig. 9. Introduction of PTFE or CPVC leads to enhancement while others results in reduction with both PVDF-PTFE and PVDF-CPVC reaching their maximum values of approximately $-0.28 \times 10^{-4} \mathrm{C} \mathrm{m}^{-2} \mathrm{~K}^{-1}$ at $R_{\min }$. Another measure for the performance of NP elastic layers is shown in Fig. 10. It demonstrates how much of an alteration potential the introduction of NP material has on the secondary PY coefficient of PVDF. The reason behind our preference for this method of comparison to that utilized in Fig. 9 is due to the fact that the secondary contribution of PVDF's PY coefficient varies quite significantly from a sample of PVDF to another owing largely to their preparation process, hence making ample assessment of alternation in secondary effect difficult. At $R_{\min }$, PVDF-Invar36 pair presents the greatest change of $960 \%$ and PVDF-St about $720 \%$, with St outperforming both $\mathrm{Al}$ and $\mathrm{Zn}$, unlike with other PY materials. Since the overall PY coefficient is negative while the secondary PY coefficient of PVDF alone under OC is positive, both Invar36 and St results in the reduction in the overall PY coefficient's magnitude. However, with negative percentile secondary contributions PTFE and CPVC leads to enhancement. At $R_{\min }$, PVDF-PTFE peaks at $-420 \%$ while PVDF-CPVC reaches $-450 \%$, both indicating over $300 \%$ increase in the secondary PY coefficient of the PVDF composites, which potentially points to extremely large enhance-

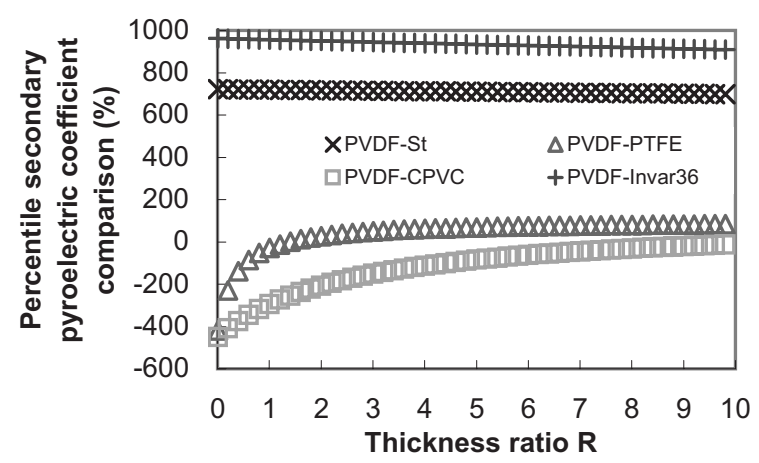

FIG. 10. Percentile secondary contribution from NP material vs thickness ratio PY to NP for PVDF pairs under OC.

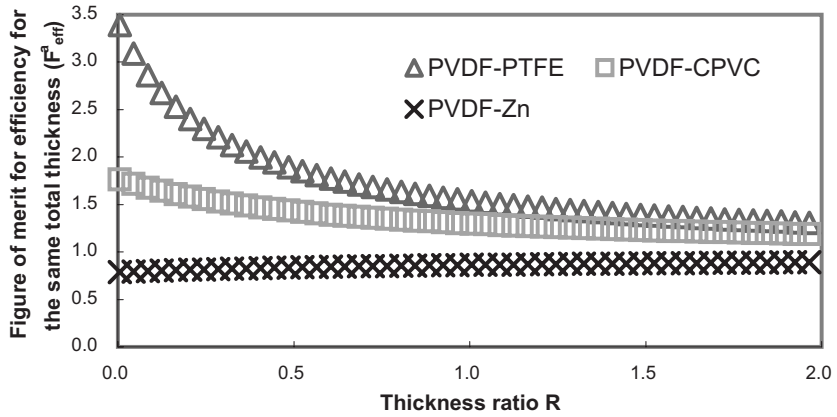

FIG. 11. Figure of merit for efficiency belonging to the same volume of PVDF composites vs thickness ratio PY to NP under OC.

ment in other PVDF samples with greater proportion of secondary contribution. Figure 11 depicts $F_{\text {eff }}^{a}$ behavior of PVDF pairs. Only PTFE and CPVC exhibit improvement in efficiency while $\mathrm{Al}$ and $\mathrm{Zn}$ nearly approach $F_{\text {eff }}^{a}=1$ mark, which St and Invar36 fail to do. At $R_{\text {min }}$, maximum $F_{\text {eff }}^{a}$ of 3.4 is attained by PVDF-PTFE, while PVDF-CPVC pair reaches 1.8 , both indicating significant improvement in efficiency.

\section{F. Best performing pairs}

In terms of the pure magnitude of the PY coefficient, PZT-5H is the best performing PY material as illustrated in Fig. 1. PZT-5H-CPVC pair's huge PY coefficient of 97 $\times 10^{-4} \mathrm{C} \mathrm{m}^{-2} \mathrm{~K}^{-1}$ at $R_{\min }$ and around $40 \times 10^{-4} \mathrm{C} \mathrm{m}^{-2} \mathrm{~K}^{-1}$ at $R=0.022$ after which PZT-5H-Zn takes over as the optimum PY coefficient pair are unrivaled by all of the PY materials investigated. As far as $F^{a}$ eff is concerned, PZT-5APTFE achieves 24, PZT-5H-CPVC 9.4, BTO-CPVC 12, and PVDF-PTFE obtains 3.4 at $R_{\min }$, while LTO-PTFE and LNO-PTFE attain 2.4 and 3.63, respectively, at $R=0.025$. PZT-5A, LTO, and PVDF all fail to register any pair that exhibited improvement in the efficiency with $F^{b}$ eff. However, PZT-5H-PTFE reaches $F_{\text {eff }}^{b}>1$ at $R>1.0$, BTO-PTFE at $R$ $>1.1$, and LNO-PTFE at $R>0.95$.

It has to be said that certain assumptions made in the thermal expansion coefficients under SC, for example, PZT manufacturer's data on coefficients of all three axes being the same, which are used to evaluate the thermal expansion coefficients under OC, make the numerical values of our secondary PY coefficients open to discussion.

\section{SUMMARY}

In summary, we have developed a mathematical model for the PY effect under OC condition and simulated 2-2 connectivity enhancement phenomena under such condition for 36 pairs of PY and NP materials. The investigated PY materials are PZT (PZT-5H and PZT-5A), BTO, LTO, LNO, and PVDF, while the NP materials are stainless steel, PTFE (Teflon), CPVC thermoplastic, aluminum, zinc, and Invar 36. On the whole, in terms of the magnitude of the PY coefficient change, $\mathrm{Zn}$ outperforms $\mathrm{Al} / \mathrm{St}$ and $\mathrm{CPVC}$ outperforms PTFE in all the pairings with PY materials except PVDF, in which case it was the opposite due to PVDF's extremely high thermal expansion coefficient. This is due to $\mathrm{Zn}$ and 
CPVC having higher thermal expansion coefficient and Young's modulus than $\mathrm{Al}$ and $\mathrm{CPVC}$, respectively. However, where the figure of merit for efficiency is concerned, this does not always hold since PTFE and Al possess lower volumetric heat capacity than $\mathrm{CPVC}$ and $\mathrm{Zn}$, respectively. Extraordinarily large PY coefficient of $97 \times 10^{-4} \mathrm{C} \mathrm{m}^{-2} \mathrm{~K}^{-1}$ at $R_{\text {min }}$ is expected for PZT-5H-CPVC while PVDF-CPVC could show increase in the secondary PY coefficient of up to $350 \%$. In addition, where the figure of merit for efficiency is concerned, for the same volume of the composite PZT-5APTFE it reaches a 24-fold increase in efficiency, while $F^{b}$ indicates in most pairings under $\mathrm{OC}$, it will struggle to achieve the same level of efficiency when additional NP materials are introduced for PY coefficient alteration.

It is clear from our analysis that there indeed is a substantial dissimilarity between the PY coefficients and figure of merit for efficiency for various PY-NP pairs under SC and OC. We believe this implies that there should be a greater distinction made between the PY coefficients under SC and OC than previously thought. Our analysis techniques provide a methodology for assessing the potentials of particular PY material and its 2-2 laminates for applications under OC condition. For instance, appraising employment credentials of LTO or LNO in applications such as PY X-ray generation, ${ }^{4-6}$ electron accelerator, ${ }^{7}$ and nuclear fusion. ${ }^{8}$

\section{ACKNOWLEDGMENTS}

We thank Professor R. W. Whatmore of Tyndall Institute, Ireland for his invaluable discussions on this work. We also thank EPSRC (Grant No. EP/D506638/1) for partial financial support.

${ }^{1}$ J. F. Nye, Physical Properties of Crystals (Oxford University Press, Oxford, 1979).

${ }^{2}$ J. van Suchtelen, Philips Res. Rep. 27, 28 (1972).

${ }^{3}$ H. H. S. Chang and Z. Huang, Appl. Phys. Lett. 92, 152903 (2008).

${ }^{4}$ J. D. Brownridge, Nature (London) 358, 287 (1992).

${ }^{5}$ J. D. Brownridge and S. Raboy, J. Appl. Phys. 86, 640 (1999).

${ }^{6}$ Y. Danon and J. A. Geuther, J. Appl. Phys. 97, 104916 (2005).

${ }^{7}$ J. D. Brownridge and S. M. Shafroth, Appl. Phys. Lett. 85, 1298 (2004).

${ }^{8}$ B. Naranjo, J. K. Gimzewski, and S. Putterman, Nature (London) 434, 1115 (2005).

${ }^{9}$ R. W. Whatmore, Rep. Prog. Phys. 49, 1335 (1986).

${ }^{10}$ Trademark of Waterloo Maple Inc., 2004.

${ }^{11}$ B. Wang and C. H. Woo, J. Appl. Phys. 100, 044114 (2006).

${ }^{12}$ M. E. Lines and A. M. Glass, Applications of Ferroelectrics and Related Materials (Oxford University Press, Oxford, 1977).
${ }^{13}$ For the elastic and thermal properties of Stainless steel-15-7PH, data provided by Goodfellow was used (2006).

${ }^{14}$ For the thermal and piezoelectric properties of PZT-5H (PSI-5H4E) and PZT-5A (PSI-5A4E), values provided by Piezo systems Inc. were used (2006).

${ }^{15}$ For the detailed mechanical properties of both PZTs, data from Ferroperm piezoceramics for the corresponding Ferroperm PZTs, namely, Pz27 for PZT-5A and Pz29 for PZT-5H, were used (2006).

${ }^{16}$ H. S. Nalwa, Ferroelectric Polymers: Chemistry, Physics, and Applications (Dekker, New York, 1995).

${ }^{17}$ Y. Xu, Ferroelectric Materials and Their Applications (North-Holland, Amsterdam, 1991)

${ }^{18}$ P. J. Grout, N. H. March, and T. L. Thorp, J. Phys. C 8, 2167 (1975).

${ }^{19}$ B. Ploss, B. Ploss, S. Kopf, and F. G. Shin, ISE 12 Proceedings, p. 487 (2005).

${ }^{20}$ R. W. Munn and R. J. Newham, J. Phys. C 7, 848 (1974).

${ }^{21}$ H. Sol, G. Roebben, W. Heylen, T. Lauwagie, Y. Shi, and O. Van der Biest, NDT \& E Int. 36, 487 (2003).

${ }^{22}$ A. E. H. Love, Mathematical Theory of Elasticity, 4th ed. (Cambridge University Press, Cambridge, 1927).

${ }^{23}$ A. G. Chynoweth, J. Appl. Phys. 27, 78 (1956)

${ }^{24}$ Landolt-Bornstein: Numerical data and functional relationships in science and technology, Group III: Crystal and Solid State Physics, edited by K.-H. Hellwege (Springer-Verlag, Berlin-Heidelberg, 1981).

${ }^{25} \mathrm{eFunda}$, Engineering fundamentals: Properties of Piezo material, (2008).

${ }^{26}$ A. W. Warner, M. Onoe, and G. A. Coquin, J. Acoust. Soc. Am. 42, 1223 (1967).

${ }^{27}$ Landolt-Bornstein: Numerical data and functional relationships in science and technology: Group III: Crystal and Solid State Physics, edited by K.-H. Hellwege (Springer-Verlag, Berlin-Heidelberg, 1982).

${ }^{28}$ R. G. Kepler and R. A. Anderson, J. Appl. Phys. 49, 4490 (1978).

${ }^{29}$ S. B. Lang and S. Muensit, Mater. Res. Soc. Symp. Proc. 889, 01.1 (2006).

${ }^{30}$ Y. Roh, V. V. Varadan, and V. K. Varadan, IEEE Trans. Ultrason. Ferroelectr. Freq. Control 49, 836 (2002).

${ }^{31}$ For the elastic and thermal properties of PTFE (Teflon), data provided by Gentech Engineering Plastics was used (2008).

${ }^{32}$ B. Scott and K. Michelle, Chem. Eng. Prog. 90, 36 (1994).

${ }^{33}$ N. Merah, M. Irfan-Ul-Haq, and Z. Khan, J. Mater. Process. Technol. 142, 247 (2003).

${ }^{34}$ For the thermal properties of $\mathrm{CPVC}, \mathrm{Al}$, and $\mathrm{Zn}$, data provided by Wikipedia Foundation Inc. was used (2008).

${ }^{35} \mathrm{eFunda}$, Engineering fundamentals: Typical properties of aluminum alloys (2008).

${ }^{36}$ For the elastic and thermal properties of $\mathrm{Zn}$, data provided by Goodfellow was used (2007).

${ }^{37}$ R. A. Paquin, Handbook of Optics, 2nd ed. (McGraw-Hill, New York, 1995).

${ }^{38}$ R. A. Paquin, Handbook of Optomechanical Engineering (CRC, Boca Raton, 1997).

${ }^{39}$ Surfacenet GmbH (2003).

${ }^{40}$ For the thermal and PY properties of LTO and LNO, values provided by Crystal Technology Inc. were used (2007).

${ }^{41}$ G. D. Sao and H. V. Tiwary, J. Appl. Phys. 53, 3040 (1982).

${ }^{42}$ D. Berlincourt, H. H. A. Krueger, and C. Near, "Properties of piezoelectricity ceramics," Report No. TP-226 2003. 